\title{
OPERACIONALIZACIÓN DE LA TAXONOMÍA DE ANDERSON Y KRATHWOHL PARA LA DOCENCIA UNIVERSITARIA
}

Carlos Bancayán Oré

\section{Resumen}

Es indispensable que el docente conozca y aplique en la gestión de la enseñanza mecanismos de ordenamiento de la información que aseguren la activación y aplicación de procesos secuenciales del pensar para garantizar la adquisición y operacionalización de conocimientos y habilidades, reflejo de competencias interiorizadas, como consecuencia de acciones estratégicas que el profesor diseña y propone en la planificación de sus sesiones de clase. Este artículo aborda la propuesta taxonómica de Lorin Anderson y David Krathwohl, en sus seis categorías del conocimiento, con las habilidades que cada una implica, las actividades que realiza el estudiante a partir de la aplicación de estrategias didácticas, propias de la educación superior, que optimizan el proceso de enseñanza-aprendizaje.

Palabras clave: Taxonomia. Taxonomia de Anderson y Krathwohl. Categorias del conocimiento. Habilidades. Estrategias metodológicas.

\section{Abstract}

It is essential that the teacher knows and applies in teaching management, mechanisms of information management to ensure activation and implementation of sequential thinking process to guarantee the acquisition and operationalization of knowledge and skills, reflexion of internalized competencies, as a result of strategic actions that the teacher designs and proposed in planning their class sessions. This article discusses the taxonomic proposal of Lorin Anderson and David Krathwohl, its six categories of knowledge, the skills that each one involves, the activities performed by students from the application of instructional strategies, distinctive of higher education, which optimize the teaching-learning process.

Key Words: Taxonomy. Anderson and Krathwohl's Taxonomy. Categories of knowledge. Skills. Methodological strategies. 
EL CONOCIMIENTO EN LA TAXONOMÍA DE ANDERSON Y KRATHWOHL

Desde mediados del siglo pasado, los educadores se han mostrado preocupados respecto a procedimientos válidos que pueden usar para planificar, implementar, ejecutar y evaluar los procesos de enseñanza-aprendizaje. Esto, a partir de contenidos curriculares que buscan asegurar la construcción, apropiación y gestión del conocimiento, de tal forma que el estudiante evidencie el uso apropiado de capacidades, competencias y actitudes en sus desempeños personales $\mathrm{y}$ profesionales.

Al respecto, se propusieron una serie de ordenamientos sistemáticos y jerárquicos de los diferentes mecanismos generadores del aprendizaje, que se denominaron taxonomías. El término taxonomía conlleva una clasificación, categorización u ordenación, que describe cómo se construye sobre lo anteriormente aprendido; refleja las diferentes formas de pensar, en una ruta diseñada por el docente, en la que trata de complejizar los procesos del pensamiento que el alumno usa para construir conocimiento.

Éste artículo aborda la exposición y aplicación práctica de la taxonomía de Anderson y Krathwohl, adaptada al campo de la educación superior; además, menciona algunas estrategias didácticas que el docente puede aplicar asociando la categoría del conocimiento con la estrategia a usar en el aula.
Anderson y Krathwohl proponen una taxonomía para procesos de enseñanza-aprendizaje y evaluación, diseñada en dos dimensiones: la del conocimiento y la de categorías del conocimiento. Esta última plantea la existencia de seis niveles: reproducción, conceptualización, aplicación, análisis evaluación y creación.

\section{REPRODUCCIÓN}

El objetivo de la instrucción es promover la retención del material en la misma forma en que fue enseñado. Cuando el profesor se concentra solo en aprendizaje memorístico la enseñanza y la evaluación se enfocan en recordar fragmentos de conocimiento, aislados del contexto.

Esto implica que el estudiante debe reconocer apropiadamente un evento o un conocimiento previo. Para ello recuerda, reproduce la información / conocimiento correcto desde la memoria, utilizando los denominados datos básicos memorizados.

Para lograr estos procesos el docente puede proponer algunas de las siguientes actividades: dirige, muestra, examina, pregunta, evalúa, dice.

Todo ello, con la finalidad de que el estudiante pueda responder, recordar, absolver, reconocer, memorizar, definir, describir, contar, etc. Por tanto, estos procesos procurarán el logro de las siguientes habilidades:

- Recuperar - Recordar, que implica: Traer conocimiento relevante de la memoria a largo plazo cuando di- 
Operacionalización de la taxonomía de Anderson y Krathwohl para la docencia universitaria

cha acción es solicitada con un requerimiento. Por ejemplo: "Nombre tres politicos y economistas precursores de la Unión Europea". "escriba la secuencia para elaborar una recensión". "recite el poema "Masa" de César Vallejo", "escriba una lista de palabras clave sobre elementos básicos del liderazgo". "reproduzca la fórmula cuadrática de la variable X", "elabore una lista con los criterios a considerar cuando cuida a un paciente con sida".

- Reconocer-Identificar, que supone: Traer conocimiento relevante de la memoria a largo plazo para compararlo con información. Por ejemplo: "identifique filósofos existencialistas en un diagrama de diferentes corrientes filosóficas". "Identifique y considere implicaciones éticas en las investigaciones científicas", "defina qué comportamientos se consideran no profesionales en la relación entre un empleador y su colaborador", "describa los procesos utilizados en la gestión del recurso humano, cuando elabore el perfil de desarrollo de los trabajadores de una empresa".

Las estrategias metodológicas que mejor se adaptan al logro de estas habilidades son: Exposición de una situación problemática (problematización) y la exposición o clase magistral.

\section{CONCEPTUALIZACIÓN}

Implica el construir significados desde la información de la enseñan- za, incluyendo la comunicación oral, escrita y gráfica. Los estudiantes comprenden cuando construyen conexiones lógicas entre el conocimiento nuevo con el previo. Por ello, el conocimiento nuevo se integra con los conocimientos previos del aprendizaje.

El estudiante debe ser capaz de aclarar, usando su propio "fraseo", lo que le representa la información recibida. Por ello, debe comprender e interpretar los datos escritos, orales, gráficos y cuantitativos. Debe construir significados a partir de materiales educativos o experiencias.

Para lograr estas situaciones, el docente procurará realizar las siguientes acciones: mostrar, escuchar, preguntar, comparar, contrastar, examinar.

Estas acciones, desarrolladas y propuestas por el docente, posibilitarán que el alumno pueda: explicar, describir, bosquejar, reiterar, traducir, mostrar, interpretar; convirtiéndose en un participante activo del proceso educativo.

Las habilidades que se logran desarrollar con este proceso son:

- Interpretar, traducir, parafrasear, representar, clarificar; los mismos que están referidos a convertir información de un tipo de representación a otra: "palabras a palabras", "palabras a imágenes", "números a palabras", "palabras a números", "notas musicales a tonos". Por ejemplo: "traduzca a una ecuación algebraica el problema que se relata", "represente mediante un diagrama el desarrollo de la iniciativa empresarial en los últimos 25 
años", "parafrasee el discurso del politico X referente a sus propuestas en educación".

- Ejemplificar, ilustrar, referir; alude a dar un ejemplo específico de un concepto o principio general. Implica, además, identificar las características de un concepto o principio y usarlas para seleccionar o construir un ejemplo o un caso, que no fue tratado durante el proceso de instrucción. Por ejemplo: "Dibuje un paralelogramo", "cite un ejemplo de cómo las técnicas de creatividad e innovación afectaron el crecimiento comercial de la industria textil en Gamarra", "represente mediante un esquema las propuestas de los candidatos presidenciales"; en el ámbito de la psicología, "esquematice las características del sindrome de burnout", etc.

- Explicar, construir modelos; implica reconocer un ejemplo o usar un modelo causa-efecto de un sistema. El modelo puede derivarse de una teoría formal, o puede ser fundamentado en la investigación o en la experiencia. Una explicación completa implica construir un modelo causa-efecto, incluyendo cada parte mayor en un sistema o cada evento mayor en una cadena y usar el modelo para determinar cómo un cambio en una parte del sistema o eslabón en la cadena, afectan un cambio en otra parte. Por ejemplo: "proporcione detalles para justificar por qué aconteció la reforma agraria peruana, cuándo y cómo sucedió", "elabore un diagrama de flujo que ilustre la secuencia de los acontecimientos del diferendo marítimo entre Chile y Perú", "explique los efectos sociales, económicos y políticos de la invasión a Irak en el mundo globalizado".

Las estrategias metodológicas que permiten lograr estas habilidades son: Exposición problémica, exposición o clase magistral, talleres de ciencias básicas, discusión en grupos pequeños, mesa redonda

\section{APLICACIÓN}

Consiste en el uso de procedimientos para realizar ejercicios o resolver problemas. Un ejercicio es una tarea para la cual el estudiante conoce el procedimiento (pasos) correcto a usar, por lo que en su uso, desarrolla una aproximación de rutina. Por ello, los problemas son tareas por las cuales el estudiante no sabe qué procedimiento usar, por lo que debe buscar y encontrar uno para la resolución del mismo.

Implica, además, que el estudiante debe utilizar un procedimiento aprendido previamente en nuevas situaciones, aplicando los conocimientos para resolver problemas.

Para lograr estos procesos, el docente puede ejecutar algunas de las siguientes actividades expresadas en su ejecución, tales como: mostrar, facilitar, observar, evaluar, organizar, preguntar. 
Estas situaciones harán que el estudiante resuelve problemas, demuestre el uso de los conocimientos, calcule, compile, complete, ilustre, construya; ello, en virtud, de ser un receptor activo.

Las habilidades que va a lograr en sus estudiantes, son:

- Ejecutar, realizar: Llevar a cabo un procedimiento cuando se confronta una tarea o ejercicio que le es familiar. El ejercicio será una secuencia de pasos con un orden determinado. Cuando los pasos se realizan correctamente, el resultado es una respuesta con coherencia y contenido lógico. Por ejemplo: "lea el siguiente pasaje de la lectura que está en inglés", "realice un tríptico que ilustre los beneficios de su carrera en su desarrollo profesional", "aplique conocimientos de control de infecciones en las instalaciones para el cuidado de pacientes", "recopile de internet fotografias de Lima de los años 50, para demostrar el crecimiento urbano de la ciudad", "relacione los cambios de energía en la ruptura y formación de enlaces", "muestre cómo los cambios en el sistema legal penal afectaron los niveles de encarcelación en el Perú de la última década”, "aplique principios de la medicina basada en evidencias para determinar diagnósticos clínicos".

- Implementar, usar: consiste en seleccionar y usar un procedimiento para realizar una tarea o proble- ma desconocido. Como ello implica seleccionar, el estudiante debe poseer un entendimiento del tipo de problema, así como el rango de procedimientos que le son posibles de acceder. Como es lógico, será necesaria alguna modificación en el procedimiento; por ello, la necesidad de asociarla a técnicas y métodos, pues no hay una respuesta predeterminada cuando el procedimiento se aplica correctamente. Por ejemplo: "diseñe un experimento para observar cómo crecen las plantas en distintos tipos de suelo", "elabore un presupuesto para realizar un trabajo de campo (encuestas y entrevistas)", “diseñe una estrategia de mercado para su producto utilizando como modelo el éxito de las variantes de la carne de avestruz", "seleccione y emplee técnicas sofisticadas para analizar las eficiencias en el uso de energía en procesos industriales complejos", "modifique las directrices en el estudio de un caso de una firma manufacturera pequeña para permitir un control de calidad de la producción más riguroso".

Las estrategias metodológicas que se pueden usar para alcanzar las capacidades de esta categoría, son: la exposición problémica, el método de la búsqueda parcial o heurística, el proyecto, los talleres de ciencias básicas, los seminarios, la discusión en grupos pequeños, la simulación y la mesa redonda. 


\section{ANÁLISIS}

Es descomponer un material en las partes que la constituyen y determinar cómo se relacionan las partes y estas, con una estructura general. Implica, por tanto, determinar las partes importantes de una información, las formas en que las partes están organizadas y el propósito inherente de la información.

El estudiante realiza lo siguiente: descompone un concepto en sus partes y describe cómo las partes se relacionan con el todo. Para ello puede, identificar las relaciones de causa efecto, las consistencias de inconsistencias, los elementos relevantes e irrelevantes de una serie de variables para llegar, finalmente, a una conclusión.

En consecuencia, el profesor realizará las siguientes acciones estratégicas: sondea, guía, observa, evalúa, actúa como un recurso, pregunta y organiza.

Esto permite que el estudiante sea un participante activo; además, explica, descubre, argumenta, debate, piensa profundamente, prueba, examina, pregunta, calcula, investiga y consulta.

Las habilidades que se logran con este nivel, son:

- Diferencia, distingue, enfoca, selecciona, discrimina, ello conlleva a distinguir las partes de una estructura total en términos de su relevancia e importancia. Es diferente al "comprender", porque implica una organización estructurada y la determinación de cómo las partes se encajan en la totalidad. Es diferente de "comparar", porque usa el contexto global para determinar qué es lo relevante y qué no lo es. Por ejemplo: "señale la información relevante en una igualdad matemática, y tache la información irrelevante", "utilice un diagrama de Venn Euler, para mostrar cómo dos temas tienen similitudes y diferencias", "mediante un diagrama muestre las relaciones entre los principales indicadores macroeconómicos", "compare y contraste los distintos modelos en el desarrollo del comercio electrónico", "compare la práctica habitual en una empresa, un psicólogo organizacional, con un administrador organizacional".

- Organizar, estructurar, integrar, encontrar coherencia, esquematizar, analizar sistemáticamente: para ello hay que identificar los elementos de una organización o situación y reconocer cómo encajan en una estructura totalizante y coherente. El estudiante construye conexiones sistemáticas y coherentes entre partes de la información que le es presentada. Por ejemplo: "haga un gráfico que ilustre los modos en que los negocios internacionales y el marketing interactúan unos con otros", "lleve a cabo una investigación para producir información que apoye la importancia de las PYMES en la economía limeña", "realice un diagrama de flujo que muestre las etapas críticas en la cadena de comercialización del 
producto X", "represente mediante un mapa mental los orígenes de dos experiencias empresariales exitosas", "calcule la pendiente en los mapas en $\mathrm{m}, \mathrm{Km}, \%$ y proporción”.

- Atribuir, deconstruir: ser capaz de establecer un punto de vista, una tendencia, valores o intenciones subyacentes a una información. Ello implica un proceso de deconstrucción en el que el estudiante determina la intención del autor de un material presentado. Para ello contrastará con "interpretar" porque el estudiante va más allá de un entendimiento básico para inferir la intención o punto de vista que subyace a la información. Por ejemplo: "lea los editoriales del diario Gestión y la Revista Perú Económico, para encontrar los puntos de vista discordantes de los autores respecto a la crisis económica europea", "determine la motivación de un personaje en una novela o cuento corto", "examine electrónicamente la propaganda de candidatos politicos, y plantee hipótesis sobre sus perspectivas en relación con diferentes problemas".

Para alcanzar estas capacidades el profesor puede usar alguna de las siguientes estrategias metodológicas: método de la búsqueda parcial o heurística, método investigativo, proyecto, talleres de ciencias básicas, seminarios, estudio de casos, discusión en grupos pequeños, resolución de problemas, mesa redonda y simposio.

\section{EVALUACIÓN}

Consiste en hacer juicios basado en criterios y estándares. Los criterios más usados son: calidad, efectividad, eficiencia y consistencia. Pueden estar determinados por el estudiante o por otros. Los estándares pueden ser cuantitativos o cualitativos y se aplican a los criterios. No todos los juicios son evaluativos, sólo los que usan estándares de desempeño con criterios claramente definidos. Esto implica que el estudiante debe "emitir juicios basados en criterios y normas", y realizar juicios basados en el resultado de un análisis. Las acciones estratégicas del docente estarán enfocadas en: aclarar, aceptar o guiar. De tal forma, que el estudiante: juzga, soluciona controversias, compara, crítica, pregunta, argumenta, evalúa, decide, selecciona y justifica.

Las habilidades que desarrollará, son:

- Revisar, examinar, detectar, monitorear, coordinar: examinar inconsistencias internas o falacias en una operación o un producto. Esto implica si una conclusión se desprende de las premisas, o si los datos avalan o anulan una hipótesis, o si el material presentado contiene partes que se contradicen unas a otras. Por ejemplo: "participe en un grupo de redacción, y retroalimente a los compañeros en cuanto a la organización y lógica de los argumentos", "escuche un discurso político y anote las contradicciones que encuentre", "revise la estructura del proyec- 
to de investigación para verificar si se incluyeron todos los pasos necesarios", "lleve a cabo un debate sobre un tema de su interés".

- Criticar-juzgar: consiste en juzgar un producto u operación basado en criterios o estándares impuestos externamente. El estudiante hace notar las características negativas y positivas de un producto $\mathrm{y}$ hace un juicio basado al menos parcialmente en esas características. El criticar está en la esencia de lo que se llama "pensamiento crítico". Por ejemplo: "juzgue en qué medida un proyecto se ajusta a los criterios de una matriz de valoración", "escriba una carta al editor de un foro o diario, y prepare una lista de criterios para juzgar", "argumente sus puntos de vista, a favor o en contra de determinado tema", "escoja el mejor método para resolver un problema matemático complejo", "determine la validez de los argumentos a favor y en contra de un tema concreto", "evalúe estrategias de marketing para diferentes modelos de comercio electrónico", "evalúe las áreas principales que contribuyen a la destreza de profesores significativos", "evalúe los niveles del proceso de enseñanza-aprendizaje que contribuyen a resolver los problemas de deserción estudiantil en las universidades".

Las estrategias metodológicas que mejor se prestan para aplicar este nivel, son:

El método de la búsqueda parcial o heurística, el método investigativo, el proyecto, los seminarios, el estudio de casos, la resolución de problemas, la mesa redonda y el simposio.

\section{CREACIÓN}

Consiste en unir elementos para formar un todo coherente o funcional. Para ello, los estudiantes hacen un nuevo producto reorganizando mentalmente algunos elementos o partes dentro de un patrón o estructura que no estaba claramente presente antes. Se coordina con las experiencias previas (saberes) de aprendizaje. Aunque demanda pensamiento creativo al estudiante, no es completamente una expresión creativa libre sin los límites que impone la tarea de aprendizaje o la situación.

Aquí, la intencionalidad de los docentes debe ser que los estudiantes sean capaces de "sintetizar material en un todo". Esta sintesis se requiere en trabajos donde se espera que el estudiante arme el material previamente enseñado en una presentación organizada.

Con esta categoria, el estudiante debe evidenciar que reúne las piezas para formar algo nuevo o reconocer los componentes de una nueva estructura. Por tanto, se trata de diseñar, construir, planificar los propios programas o alternativas para satisfacer necesidades, o llegar a objetivos concretos, generando hipótesis y creando una estructura completamente nueva.

En este nivel, el docente es un facilitador del proceso, donde el 
estudiante: diseña, formula, planifica, toma riesgos, modifica, crea y propone.

Las habilidades que se conseguirán son:

- Generar-Conjeturar: consiste en representar el problema y llegar a las alternativas o hipótesis que cumplan ciertos criterios. Con frecuencia, la forma en que el problema se representa inicialmente sugiere posibles soluciones; sin embargo, redefinir o sacar una nueva representación del problema puede sugerir soluciones diferentes. Por ejemplo: "con base en una lista de criterios, escriba algunas opciones para mejorar las relaciones laborales en las empresas peruanas", "genere diversas hipótesis científicas para explicar las causas de las crisis económicas recurrentes del capitalismo", "cree un producto, dele un nombre y plantee una campaña de marketing de posicionamiento del mismo", "proponga un grupo de alternativas para reducir la dependencia de combustibles fósiles, que contemple tanto aspectos de interés económico como ambiental", "sugiera hipótesis alternativas, basadas en los criterios que expresan los indicadores", "venda una idea".

- Planear-Diseñar: consiste en concebir una solución que cumpla los criterios del problema, esto es, desarrollar un plan para resolver el problema. Se trata por tanto, de planificar con la finalidad de llevar a cabo los pasos para crear la solución de un problema. Un estudiante puede establecer submetas o sub-tareas para ejecutarlas mientras se soluciona el problema desde la perspectiva de acción de sus partes. Por ejemplo: "esboce un trabajo de investigación sobre el comportamiento organizacional desde un punto de vista de responsabilidad social", "diseñe un estudio científico para probar el efecto de los distintos tipos de música en la cultura popular peruana", "reconozca y formule problemas que son susceptibles para solucionar los problemas de estrés laboral y cansancio de obreros en diferentes rubros de la producción".

- Producir-Construir: consiste en llevar a cabo un plan para resolver un problema dado que cumpla ciertas especificaciones (características). Puede incluir o no originalidad o singularidad como una de las especificaciones. Por ejemplo: "escriba un reporte de situaciones diarias desde el punto de vista de un gestor en Recursos Humanos", "proponga soluciones en forma oral y en forma escrita para solucionar problemas de manejo energético que sean complejos".

Las estrategias metodológicas que mejor se adaptan a este nivel, son: el método de la busqueda parcial o heurística, el método investigativo, el proyecto, los seminarios, el estudio de casos, la resolución de problemas, y el simposio. 


\section{CONCLUSIONES}

- Es Indispensable, por parte del docente universitario, el conocimiento y aplicación de taxonomías que le posibiliten que pueda planificar la activación, ordenamiento y aplicación de procesos mentales simples y complejos en sus estudiantes, de tal forma que se pueda garantizar la adquisición de capacidades, la aplicación de habilidades y actitudes, y la evidencia de ser competente.

- La taxonomía de Anderson y Krathwohl es una secuencia ordenada y lógica que permite al docente una visión compleja de las habilidades que desea alcanzar en sus estudiantes, asociado a acciones concretas que ellos pueden realizar en el aula o fuera de ella, como consecuencia de directrices ad hoc propuestas por el profesor.

- Las categorías mentales que requieren menor esfuerzo mental son: reproducción, conceptualización y aplicación; las de mayor complejidad y que deben ser usadas intensivamente en la formación profesional son: análisis, evaluación y creación.

- Se evidencia que a mayor nivel de complejidad en las categorias, la participación del estudiante es mayor, más profunda y comprometida en la construcción de su conocimiento, vía la utilización de herramientas mentales o materiales; por lo tanto, son las categorias, más complejas, las que requieren de mayor esfuerzo mental en el sentido de ser las que garantizan la adquisición y aplicación de competencias.

- La manipulación de información y su transformación en conocimiento está asociada a ciertas estrategias metodológicas, propias de la educación superior, que el docente debe aplicar para garantizar su logro. 
Operacionalización de la taxonomía de Anderson y Krathwohl para la docencia universitaria

\section{REFERENCIAS}

ANDERSON, w. 1. y David r. KRATHWOHL, D. r., et al (Eds.). (2001) Una taxonomía para el aprendizaje, enseñanza y evaluación: una revisión de la taxonomía de Bloom de objetivos educativos. Boston: Allyn \& Bacon.

BOSHELL, M. (2007). Dinámicas e investigación en las redes de educación superior. Colombia: Universidad Santo Tomás.

CHURCHES, A. (2009). Taxonomía de Bloom para la era digital. Recuperado de http://www.eduteka.org/TaxonomiaBloomDigital.php

DIRECCIÓN DE INVESTIGACIÓN Y DESARROLLO EDUCATIVO, Vicerrectoría Académica, Instituto Tecnológico y de Estudios Superiores de Monterrey. (2005). Las estrategias y técnicas didácticas en el rediseño. Recuperado de: http:/ / www. uctemuco.cl/cedid/archivos/apoyo/Capacitacion\%20en\%20estrategias $\% 20$ y\%20tecnicas\%20didacticas.pdf

Educational Origami. (mayo 2011). Revisión de la taxonomía de Bloom. Recuperado de http://edorigami.wikispaces.com/Bloom's+Digital+Taxonomy

GARCÍA, J. (1998). Desarrollo y conocimiento. España: Siglo XXI.

IOWA STATE UNIVERSITY. (2011). Un modelo de aprendizaje por objetivos. Recuperado de http://www.celt.iastate.edu/teaching/RevisedBlooms1.html

LAGO, B., COLVIN, L. y CACHEIRO, M. (octubre de 2008). Estilos de aprendizaje y actividades polifásicas: Modelo EAAP. Revista estilos de Aprendizaje, 2 (2), $2-22$.

OWEN, L. (2006). Más allá de Bloom- Una nueva versión de la taxonomía cognitiva. Recuperado de http://www4.uwsp.edu/education/lwilson/curric/ newtaxonomy.htm

MENDOZA, P. (1996). El debate en el aula. Madrid: Ediciones pedagógicas.

RLICH, Donald et al. (2004). Técnicas de enseñanza, México: Limusa.

WOOLFOK, A. (2007). Psicología Educativa (9a. ed.). México: Pearson Educación de México. 